\title{
Tamanho de primeira maturação para cinco espécies de peixes em uma área da
}

\section{Costa Amazônica Maranhense, Brasil}

\author{
First maturation size for five species of fish in an area of the Amazon Coast Maranhense, Brazil \\ Primer tamaño de maduración de cinco especies de peces en una zona de la Costa Amazónica de \\ Maranhense, Brasil
}

Recebido: 20/02/2021 | Revisado: 25/02/2021 | Aceito: 06/03/2021 | Publicado: 14/03/2021

\author{
Ádila Patrícia Chaves Silva \\ ORCID: https://orcid.org/0000-0003-0166-1057 \\ Universidade Estadual do Maranhão, Brasil \\ E-mail: adilachaves@gmail.com \\ Irayana Fernanda da Silva Carvalho \\ ORCID: https://orcid.org/0000-0002-4971-9804 \\ Universidade Estadual do Maranhão, Brasil \\ E-mail:nanda.dih@live.com \\ Ana Luiza Caldas Diniz \\ ORCID: https://orcid.org/0000-0003-2701-566X \\ Universidade Estadual do Maranhão, Brasil \\ E-mail: luizadiniz13@hotmail.com \\ Lorrane Gabrielle Cantanhêde \\ ORCID: https://orcid.org/0000-0001-7796-9416 \\ Universidade Federal do Pará, Brasi \\ Museu Paraense Emílio Goeldi, Brasil \\ E-mail: lorranegabrielle@hotmail.com \\ Zafira da Silva de Almeida \\ ORCID: https://orcid.org/0000-0002-8295-5040 \\ Universidade Estadual do Maranhão, Brasil \\ E-mail: zafiraalmeida@gmail.com
}

\begin{abstract}
Resumo
O presente estudo objetivou determinar os tamanhos de primeira maturação sexual de cinco espécies comercias de uma área da Costa Amazônica Maranhense, para serem utilizados como indicador no ordenamento pesqueiro na região. O comprimento de primeira maturação gonadal (L50) das espécies: Bagre bagre, Lutjanus synagris, Macrodon ancylodon, Mugil curema e Trichiurus lepturus foi estimado a partir da curva acumulada de frequências de ocorrência de indivíduos adultos por classe de comprimento total ajustado à função logística. Os comprimentos de primeira maturação gonadal de B. bagre, L. synagris, M. ancylodon, M. curema e T. lepturus foram $24,28 \mathrm{~cm}, 26,52$ $\mathrm{cm}, 19,54 \mathrm{~cm}, 19,93 \mathrm{~cm}$ e $51,45 \mathrm{~cm}$, respectivamente. Assim, sugere-se evitar a captura, armazenamento, desembarque e venda de indivíduos menores que os valores apresentados neste estudo.
\end{abstract}

Palavras-chave: Gestão pesqueira; Ictiologia; Reprodução.

\begin{abstract}
The present study aimed to determine the sizes of first sexual maturation of five commercial species of an area of the Maranhense Amazon Coast, to be used as an indicator in the fishing order in the region. The first gonadal maturation length (L50) of species: Bagre bagre, Lutjanus synagris, Macrodon ancylodon, Mugil curema and Trichiurus lepturus was estimated from the cumulative curve of frequencies of occurrence of adult individuals by class of total length adjusted to the logistic function. The first gonadal maturation lengths of B. bagre, L. synagris, M. ancylodon, M. curema and T. lepturus were $24.28 \mathrm{~cm}, 26.52 \mathrm{~cm}, 19.54 \mathrm{~cm}, 19.93 \mathrm{~cm}$ and $51.45 \mathrm{~cm}$, respectively. Thus, it is suggested to avoid capturing, storage, disembarkation and sale of individuals smaller than the values presented in this study.
\end{abstract}

Keywords: Fisheries management; Ichthyology; Reproduction.

\section{Resumen}

El presente estudio tuvo como objetivo determinar los tamaños de primera maduración sexual de cinco especies comerciales de un área de la Costa Amazónica Maranhense, para ser utilizado como indicador en la planificación pesquera en la región. La primera longitud de maduración gonadal (L50) de espécies: Bagre bagre, Lutjanus synagris, Macrodon ancylodon, Mugil curema y Trichiurus lepturus se estimó a partir de la curva acumulada de frecuencias de ocurrencia de individuos adultos por clase de longitud total ajustada a la función logística. Las primeras longitudes de 
maduración gonadal de B. bagre, L. synagris, M. ancylodon, M. curema e T. lepturus fueron $24,28 \mathrm{~cm}, 26,52 \mathrm{~cm}$, $19,54 \mathrm{~cm}, 19,93 \mathrm{~cm}$ y $51,45 \mathrm{~cm}$, respectivamente. Por tanto, se sugiere evitar capturar, almacenamiento, desembarco y venta de individuos menores a los valores presentados en este estudio.

Palabras clave: Ordenación pesquera; Ictiología; Reproducción.

\section{Introdução}

O Maranhão apresenta clima tropical, uma diversificada vegetação e inúmeras áreas de reentrâncias, caracterizadas por abranger uma grande extensão litorânea no estado, formando várias ilhas. Por conseguinte, as reentrâncias maranhenses formam uma área de proteção ambiental de uso sustentável da natureza, nas quais a pesca se torna uma das principais atividades extrativas. $\mathrm{O}$ estado que possui a segunda maior costa litorânea do Brasil, apresenta condições favoráveis, devido suas características ambientais, como grande aporte de matéria orgânica carreada por seus inúmeros rios e extensa faixa de manguezal que favorece o desenvolvimento de populações de espécies marinhas (Ferreira et al., 2014). A produção e comercialização de pescados no estado ocorrem, principalmente, por meio da pesca artesanal, atividade responsável pelo sustento de várias famílias (Santos et al., 2017).

Entre os municípios maranhenses, a Raposa, possui destaque na produção pesqueira (Cepene, 2007), por apresentar a maior e mais desenvolvida comunidade pesqueira do estado. Um dos fatores que favorecem seu destaque no setor pesqueiro é a autonomia pesqueira, considerada a maior do estado (Almeida et al., 2006), ou seja, a pesca realizada nesta região, apesar de caracterizada como artesanal de pequena escala, possibilita aos pescadores um esforço de pesca com mais tempo de navegação por viagem, conquistando maior êxito nas pescarias. Outro fator importante é a localização geográfica do município que possibilita que a região funcione como grande escoadouro de produtos oriundos da pesca (Soares et al., 2006; Almeida, 2009).

Diante disso, os pescadores artesanais vêm sofrendo certos problemas econômicos, principalmente, em decorrência da escassez e exploração desordenada, o que pode ocasionar uma sobrepesca na região (Almeida et al., 2016). Com isso, conhecer a biologia reprodutiva dos peixes é uma forma de contribuir para a compreensão de sua dinâmica populacional, podendo assim oferecer subsídios às medidas de ordenamento pesqueiro (Dala-Corte; Azevedo, 2010).

Em termos de restrições de pesca, as legislações aplicam dois instrumentos de política primária, uma temporada de pesca fechada (defeso) e limites de tamanho de captura para algumas espécies (Senguttuvan; Sivakumar, 2016). Para implementação destes dois instrumentos, as informações acerca da biologia reprodutiva das espécies se tornam necessária.

Dessa forma, objetivou-se caracterizar os tamanhos mínimos de Bagre bagre, Lutjanus synagris, Macrodon ancylodon, Mugil curema e Trichiurus lepturus, espécies de importância comercial pertencentes a uma área da Costa Amazônica Maranhense, com vista a sustentabilidade das espécies por meio de programas de manejo e elaboração da regulamentação de pesca.

\section{Metodologia}

\section{1 Área de estudo}

Esse estudo foi realizado em uma área da Costa Amazônica Maranhense, no munícipio de Raposa, Maranhão (Figura 1), localizado a nordeste de São Luís, a oeste da baía de São Marcos e a leste da baía de São José, cujas coordenadas geográficas são $02^{\circ} 25^{\prime \prime} 14.7^{\prime}$ S e $44^{\circ} 07^{\prime} 25.1$ ” W (Santos et al., 2011). 
Figura 1. Mapa de localização do município de Raposa, localizado na Costa Amazônica Maranhense, Brasil.

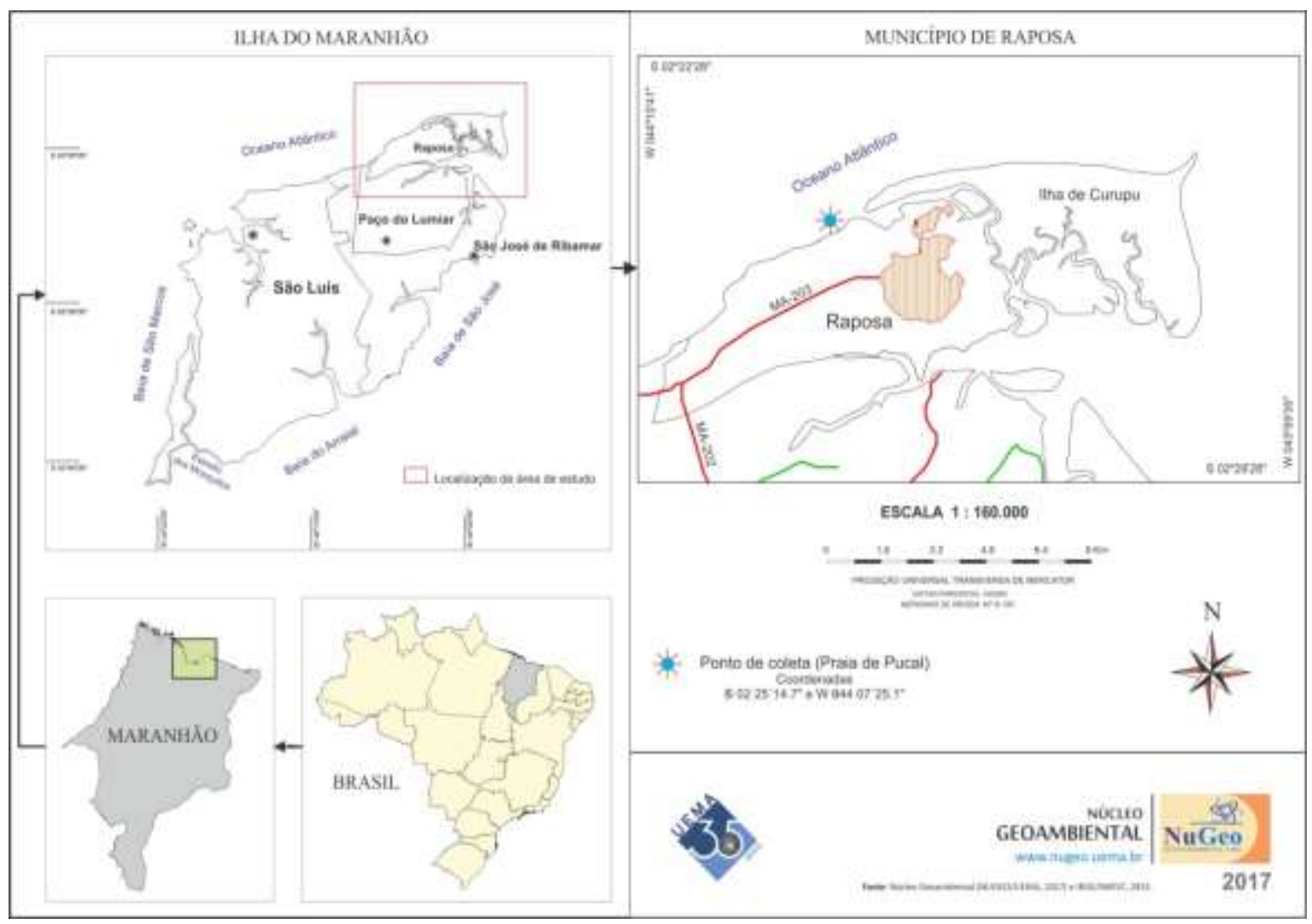

Fonte: Núcleo Geoambiental (NUGEO/UEMA, 2017).

\subsection{Análise dos dados}

Foram considerados informações de biologia reprodutiva de indivíduos de Bagre bagre, Lutjanus synagris, Macrodon ancylodon, Mugil curema e Trichiurus lepturus. Estes dados foram adquiridos por meio da compilação de dados pretéritos coletados nos anos de 2012 a 2016, licenciado pelo SISBIO № 51437.

Os exemplares foram adquiridos por meio da pesca experimental e pesca comercial, ambas realizadas de forma artesanal. As amostras foram acondicionadas em gelo e transportadas para o laboratório de Pesca e Ecologia Aquática LabPea, da Universidade Estadual do Maranhão.

As espécies foram identificadas por chaves e guias de identificação de peixes marinhos da região nordeste (Nóbrega et al., 2009); Peixes marinhos do Brasil: guia prático de identificação (Szpilman, 2000); Catálogo das espécies de peixes marinhos do Brasil (Menezes et al, 2003).

Para identificação do sexo e determinação dos estádios maturacionais, adotou-se a terminologia proposta por Brown Petterson et al. (2011) e Lowerre-Barbieri et al. (2011), adaptando para a seguinte classificação: Fase imatura (FI); Fase de desenvolvimento (FD); Fase de capacidade de desova (FCD); Fase de regressão (FR); Fase de regeneração (FRG).

A proporção sexual usada para a composição da população foi calculada para o período total e por classes de comprimento. As proporções sexuais encontradas foram testadas pelo $\chi^{2}$ (qui-quadrado) com nível de significância de $5 \%$ para verificar a existências significativas na proporção entre os sexos.

O comprimento de primeira maturação gonadal (L50) foi estimado a partir da curva acumulada de frequências de ocorrência de indivíduos adultos por classe de comprimento total ajustado à função logística (King, 1996). O L50 indica o comprimento em que $50 \%$ dos indivíduos estão aptos a reproduzir. Os estádios de maturação foram agrupados em: imaturos (fase imatura) e maturos (fase de desenvolvimento, fase de capacidade de desova, fase de regressão e fase de regeneração). O 
percentual de maturos por classe de comprimento foi calculado e considerando como variável dependente (Y) e o comprimento total como variável independente (X). Posteriormente, estes valores foram ajustados a uma curva logística, utilizando o Programa Statistica 7.0 segundo a fórmula: $\mathrm{P}=1 /(1+\exp [-\mathrm{r}(\mathrm{L}-\mathrm{Lm})])$, em que $\mathrm{P}$ é a proporção de indivíduos maduros, $\mathrm{r}$ é o declive da curva, L é o comprimento e o Lm é o comprimento médio de maturidade sexual ou o comprimento que corresponde para uma proporção de 0.5 (ou $50 \%$ ) em condições reprodutivas.

\section{Resultados e Discussão}

Foram analisados dados de um total de 1.230 espécimes. A espécie mais representativa nas capturas foi T. lepturus, sendo as fêmeas responsáveis pela maior representatividade (Tabela 1). O valor de $\chi^{2}(\mathrm{GL}: 1 ; \mathrm{p}<0,05)$ confirma a diferença significativa entre os sexos para todas as espécies.

Tabela 1. Proporção sexual, percentual e valores do qui-quadrado $\left(\chi^{2}\right)$ de fêmeas e machos por espécie em uma área da costa amazônica maranhense, no período de dezembro de 2012 a dezembro de 2016.

\begin{tabular}{ccccccc}
\hline Espécies & Fêmeas & $\%$ & Machos & $\%$ & $\begin{array}{c}\text { Proporção } \\
\text { sexual }\end{array}$ & $\chi^{2}$ \\
\hline Bagre bagre & 136 & 70.11 & 58 & 29,89 & $2.3 \mathrm{~F}: 1 \mathrm{M}$ & 31,36 \\
Lutjanus synagris & 167 & 69.66 & 71 & 30.34 & $2.9 \mathrm{~F}: 1 \mathrm{M}$ & 36,17 \\
Macrodon ancylodon & 167 & 70.47 & 70 & 29.53 & $2.3 \mathrm{~F}: 1 \mathrm{M}$ & 39,70 \\
Mugil curema & 113 & 66.09 & 58 & 33.91 & $1.9 \mathrm{~F}: 1 \mathrm{M}$ & 17,69 \\
Trichiurus lepturus & 315 & 79.95 & 79 & 20.05 & $3.9 \mathrm{~F}: 1 \mathrm{M}$ & 141,36 \\
\hline
\end{tabular}

Fonte: Autores.

A proporção sexual com predomínio de fêmeas encontradas para as espécies não diferiu do encontrado para as mesmas espécies em outras regiões do país, como por exemplo, para T. lepturus em Bitupitá, extremo oeste do Ceará (Silva, 2019), para L. synagris no Rio grande do Norte (Lira, 2019) e para B. bagre na Ilha de Cururupu, Maranhão (Véras \& Almeida, 2016). Essa variação na proporção sexual entre populações de uma mesma espécie pode ser atribuída a fatores como a pesca, sazonalidade e segregação na formação de cardumes em áreas de alimentação e desova (Santos et al., 2015).

Analisando a distribuição da frequência percentual por estágios maturacionais para o período total de estudo, observase que as cinco espécies apresentaram apenas indivíduos em fase imatura nas menores classes de comprimento, desenvolvendo a maturação à medida em que os intervalos das classes de comprimento aumentam. Para todas as espécies foi possível observar que a frequência de machos e fêmeas, quanto ao tamanho em que foram capturados, diminui à medida em que se aproxima dos maiores intervalos de tamanho (Figura 2). 
Figura 2. Distribuição da frequência percentual por classes de comprimento por estádios de maturação e para os sexos, (a) Bagre bagre, (b) Lutjanus synagris, (c) Macrodon ancylodon, (d) Mugil curema, (e) Trichiurus lepturus em uma área da costa amazônica maranhense, no período de dezembro de 2012 a dezembro de 2016. * IM (Fase imatura), FD (Fase em desenvolvimento), FCD (Fase de Capacidade de desova), FR (Fase de regressão).
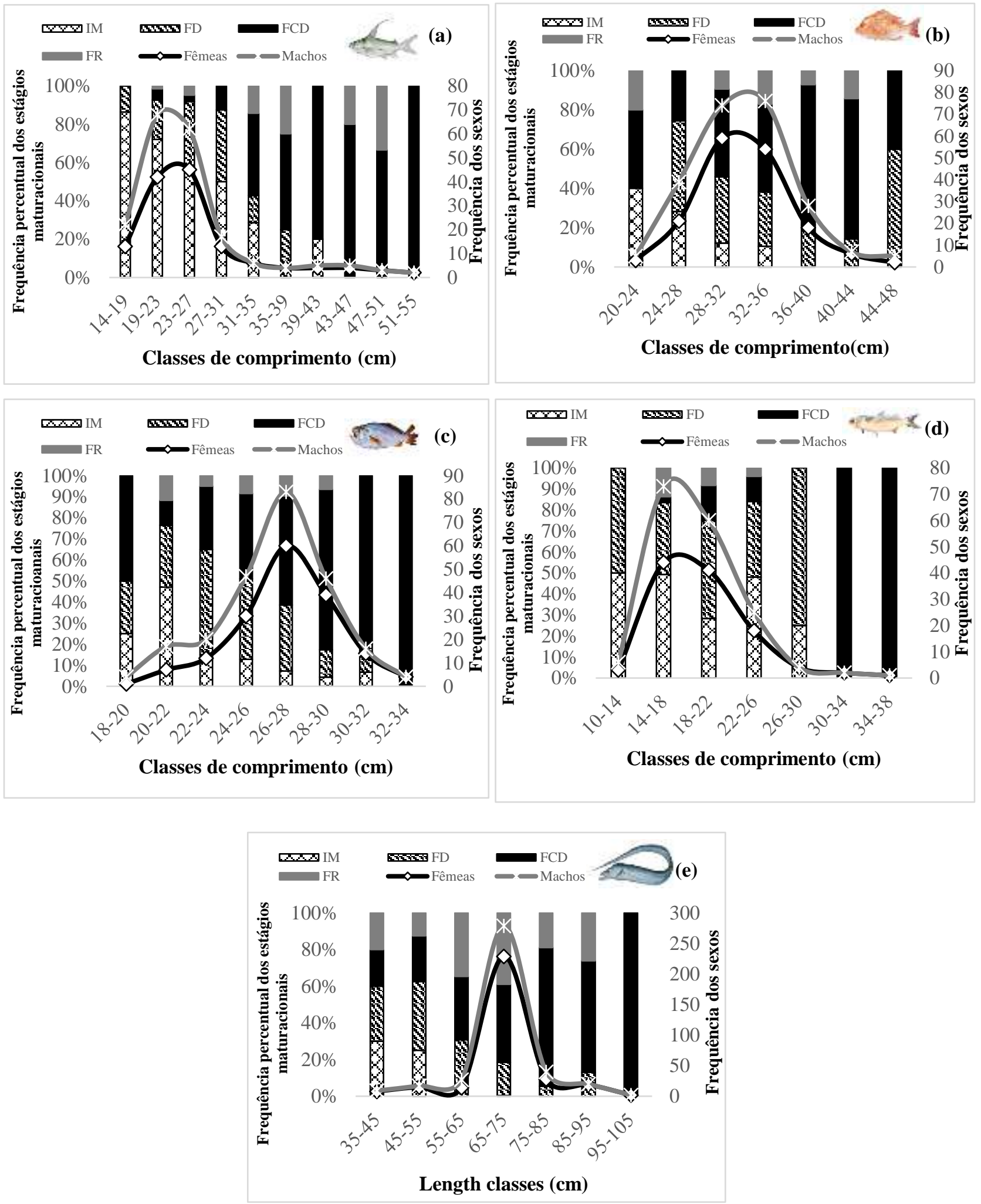

Fonte: Autores. 
Em relação ao comprimento de primeira maturação gonadal para fêmeas e machos, observa-se que os machos entram em maturação em tamanhos menores que as fêmeas (Tabela 2). Isto representa um padrão comum observado entre os peixes, não sendo recomendado sua utilização para a gestão pesqueira (Tsikliras \& Stergiou, 2014).

Tabela 2. Comprimento de primeira maturação gonadal para fêmeas e machos, por espécie em uma área da costa amazônica maranhense, no período de dezembro de 2012 a dezembro de 2016.

Comprimento total $(\mathrm{cm})$

\begin{tabular}{lccccc}
\hline Espécies & Sexo & Média \pm DP & Mínimo & Máximo & L50 \\
\hline Bagre bagre & $\mathrm{F}$ & $25,95 \pm 7,9$ & 14,2 & 54,5 & 23,02 \\
& $\mathrm{M}$ & $22,72 \pm 4,9$ & 17,3 & 46,6 & 21,87 \\
Lutjanus synagris & $\mathrm{F}$ & $31,84 \pm 4,2$ & 20 & 22 & 25,42 \\
& $\mathrm{M}$ & $31,26 \pm 5,2$ & 48 & 48 & 23,99 \\
Macrodon ancylodon & $\mathrm{F}$ & $26,59 \pm 2,6$ & 19 & 33,5 & 22,08 \\
& $\mathrm{M}$ & $24,92 \pm 2,8$ & 18,2 & 32 & 19,72 \\
Mugil curema & $\mathrm{F}$ & $19,31 \pm 3,9$ & 10,5 & 37 & 21,04 \\
& $\mathrm{M}$ & $17,81 \pm 3,0$ & 10,5 & 25 & 18,50 \\
Trichiurus lepturus & $\mathrm{F}$ & $72,26 \pm 9,6$ & 35 & 35,9 & 55,51 \\
& $\mathrm{M}$ & $68,56 \pm 7,8$ & 104,5 & 87,3 & 52,50 \\
\hline
\end{tabular}

Fonte: Autores.

Os comprimentos de primeira maturação gonadal indicados nos gráficos podem ser estabelecidos como os comprimentos médios, uma vez que utiliza o agrupamento de ambos os sexos. Os comprimentos médios de primeira maturação gonadal foram: para B. bagre de 24,28 cm, para L. synagris de 26,52 cm, para M. ancylodon de 19,54 cm, para M. curema de 19,93 cm e para T. lepturus de 51,46 cm (Figura 3). 
Figura 3. Comprimentos estimados de maturação sexual, L50 (comprimento mínimo). (a) Bagre bagre, (b) Lutjanus synagris, (c) Macrodon ancylodon, (d) Mugil curema, (e) Trichiurus lepturus em uma área da costa amazônica maranhense, no período de dezembro de 2012 a dezembro de 2016.
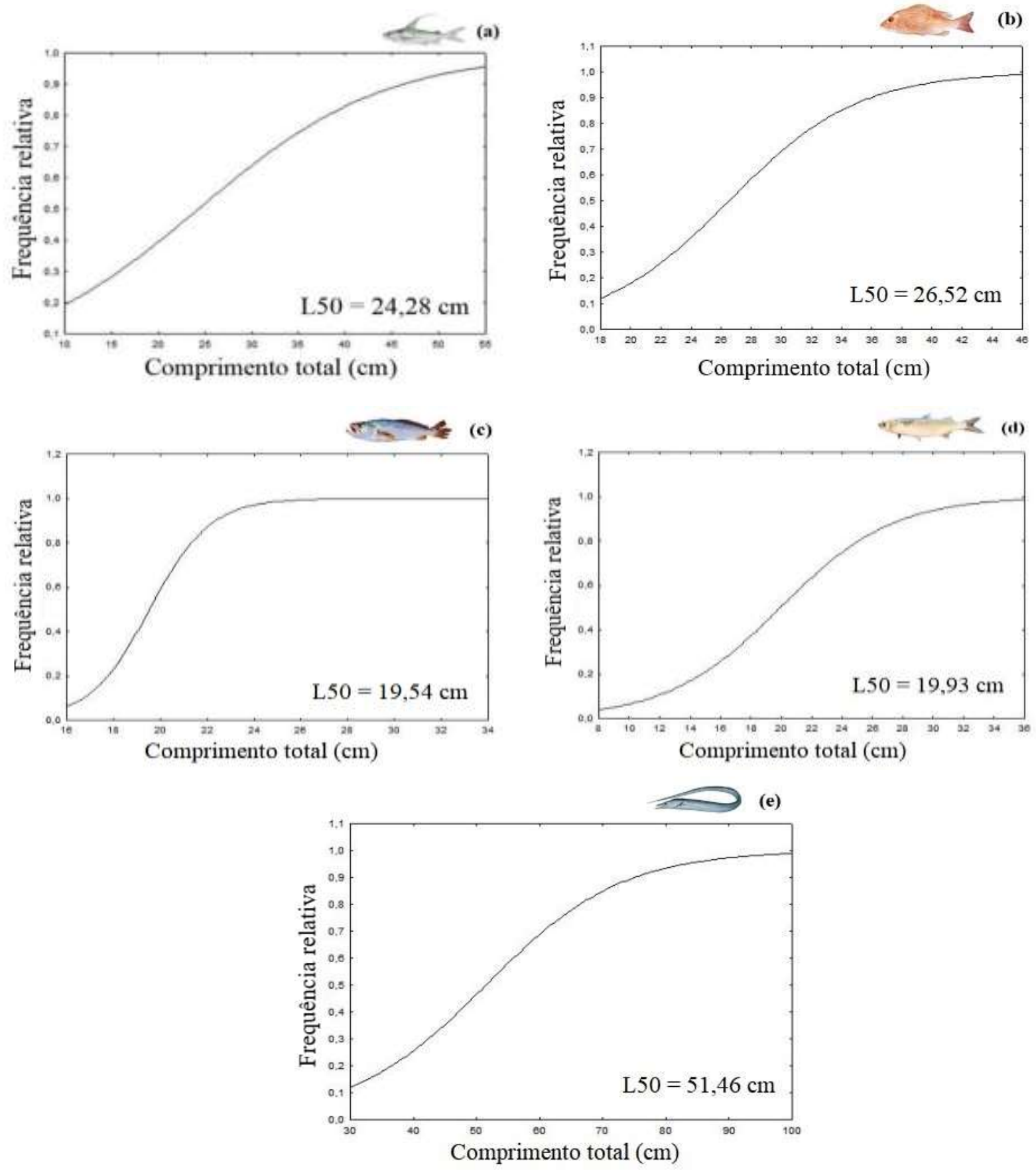

Fonte: Autores.

Resultados semelhantes foram encontrados para B. bagre, em um estudo realizado por Freitas (2020) na região da Raposa, Maranhão, onde a espécie atingiu a primeira maturação com $24,1 \mathrm{~cm}$. Por outro lado, valores maiores foram registrados para L. synagris em Abrolhos, onde a primeira maturidade ocorreu entre $23 \mathrm{~cm}$ e $24 \mathrm{~cm}$ (Freitas et al., 2014). Para M. curema, o comprimento estimado foi de 24,86 no litoral do estado de São Paulo (Fernandez \& Dias, 2013), para M. 
ancylodon na Zona Costeira de Buenos Aires, Argentina, o tamanho de primeira maturação foi de 21,1 cm (Militelli et al., 2013) e Nunes (2020) registrou para T. lepturus, em Raposa, Maranhão, o comprimento de 60,37 cm.

O comprimento de primeira maturação pode apresentar variações dependendo da disponibilidade de alimento, apresentando assim, em diferentes regiões, taxas de crescimento diferenciadas (Santos et al., 2015). Segundo Vazzoler (1996), o L50 está intimamente relacionado ao crescimento, apresentando variações intraespecíficas relacionadas a condições ambientais abióticas e bióticas.

Para a política de gestão pesqueira o comprimento de primeira maturação gonadal (L50) é um parâmetro extremamente importante na manutenção de populações de peixes exploradas comercialmente (Santos et al., 2015). No entanto, no Maranhão, a Portaria IBAMA n ${ }^{\circ} 85 / 2003$ estabelece apenas o período de defeso da fauna aquática no período de $1^{\circ}$ de dezembro a 30 de março (IBAMA, 2003) para as bacias hidrográficas genuinamente maranhenses. Contudo, essa portaria não inclui as espécies marinhas e nem a região estudada.

A legislação em vigor em Santa Catarina (Instrução Normativa MMA n n 53, de 22 de novembro de 2005; Brasil, 2005) estabelece o comprimento mínimo de captura semelhante ao encontrado nesse estudo para M. curema, com tamanho de $20 \mathrm{~cm}$, porém diverge dos tamanhos encontrados para M. ancylodon e para T. lepturus, que a instrução normativa registra os comprimentos de $25 \mathrm{~cm}$ e $70 \mathrm{~cm}$, respectivamente.

A remoção de espécies em tamanhos menores que o permitido pela legislação pode ser prejudicial para as unidades populacionais de crescimento lento e maturação tardia (Froese et al. 2008), pois são retiradas do ambiente antes de desenvolver ao menos um ciclo reprodutivo (Beverton \& Holt, 1957).

\section{Conclusão}

Sugere-se que sejam criadas medidas de manejo pesqueiro para a Costa Amazônica Maranhense, que respeitem as normas para tamanhos mínimos de captura. Recomenda-se através deste estudo que seja evitada a captura, armazenamento, desembarque e venda de B. bagre, L. synagris, M. ancylodon, M. curema e T. lepturus, menores que: 24,28 cm, 26,52 cm, $19,54 \mathrm{~cm}, 19,93 \mathrm{~cm}$ e $51,45 \mathrm{~cm}$, respectivamente.

\section{Referências}

Almeida, Z. S. Os recursos pesqueiros marinhos e estuarinos do Maranhão: Biologia, Tecnologia, Estado da Arte e Manejo. Tese de doutorado. Universidade Federal do Pará, 2009.

Almeida, Z. S., Castro, A. C. L., Paz, A. C., Ribeiro, D., Santos, N. B., \& Ramos, T. Diagnóstico da pesca artesanal no litoral do estado do Maranhão. In: Isaac, V. J., Martins, A. S., Haimovici, M., \& Andriguetto Filho, J. M. (Ed.). A pesca marinha e estuarina do Brasil no início do século XXI: recursos, tecnologias, aspectos socioeconômicos e institucionais. Belém: Pará, p.41-65, 2006.

Almeida, Z. S., Santos, N. B., Sousa, H. L., Carvalho Neta, R. N. F., \& Andrade, T. S. O. M. Biologia reprodutiva da pescada amarela (Cynoscion acoupa) capturada na baía de São Marcos, Maranhão, Brasil. Biota Amazônia. Macapá, 6(1), 46-54, 2016.

Beverton, R. J. H., \& Holt, S. J. On the dynamics of exploited fish populations. UK ministry of agriculture, fisheries and food, ser 2 , vol 19 , London. 1957.

Brown-Peterson, N. J., Wyanski, D. M., Saborido-Rey, F., Macewicz, B. J., \& Lowerre-Barbieri, S. K. A Standardized Terminology for Describing Reproductive Development in Fishes. Marine and Coastal Fisheries: Dynamics, Management, and Ecosystem. Science 3 (1): $52-70.2011$.

Cepene, Boletim estatístico da pesca marítima e estuarina do Nordeste do Brasil 2005. Brasília, DF: Ministério do Meio Ambiente, Instituto Brasileiro do Meio Ambiente e dos Recursos Naturais Renováveis, Centro de Pesquisa e Gestão de Recursos Pesqueiros do Litoral Nordeste. 211 p. 2007.

Dala-Corte, R. B., \& Azevedo, M. A. Biologia reprodutiva de Astyanax henseli (Teleostei, Characidae) do curso superior do rio dos Sinos, RS, Brasil. Iheringia Série Zoologia, 100(3): 259-266. 2010.

Fernandez, W. S., \& Dias, J. F. Aspectos da reprodução de Mugil curema Valenciennes, 1836 em dois sistemas costeiros do sudeste do Brasil. Tropical Zoology, 26(1), 15-32, 2013.

Ferreira, I. S., Cutrim, M. V. M., Watanabe, I. S., \& Mendonça, E. M. C. O Município de Raposa - MA: do abandono ambiental as perspectivas de crescimento econômico e turístico. VII Congresso Brasileiro de Geógrafos, Vitória/ES, 2014. 
Freitas, J. Estimativa do período reprodutivo e tamanho de primeira maturação de peixes comercialmente importantes no litoral do estado do Maranhão: uma ferramenta para a gestão pesqueira. Dissertação. Universidade Estadual do Maranhão, 72 p. 2020.

Freitas, M. O., Rocha, G. R. A., Chaves, P. T. C., \& Moura, R. L. Reproductive biology of the lane snapper, Lutjanus synagris, and recommendations for its management on the Abrolhos Shelf, Brazil. Journal of the Marine Biological Association of the United Kingdom, 94 (8), 1711-1720, 2014.

Froese, R., Stern-Pirlot, A., Winker, H., \& Gascuel, D. Size matters: how single species management can contribute to ecosystem-based fisheries management. Fisheries Research, 92: 231-241. 2008.

Instituto Brasileiro do Meio Ambiente e dos Recursos Naturais Renováveis (IBAMA). Portaria $n^{\circ} 85$, de 31 de dezembro de 2003. <http://www.icmbio.gov.br/cepsul/images/stories/legislacao/ Portaria/2003/pibama852003defesobaciashidrograficasmavigindopibama92004.pdf>

King, M. Fisheries biology: assessment and managment. Fishing News Books. 341 p. 1995.

Lira, R. D. Dinâmica populacional do peixe ariocó, Lutjanus synagris (Linnaues, 1758) (Actinopterygii; Perciformes) nas águas costeiras do Rio Grande do Norte, Brasil. Universidade Federal do Rio Grande do Norte. 54 p. 2019.

Lowerre-Barbieri, S. K., Brown-Peterson, N. J., Murua, H., Tomkiewicz, J., Wyanski, D., \& Saborido-Rey, F. Emerging issues and methodological advances in fisheries reproductive biology. Marine and Coastal Fisheries: Dynamics, Management, and Ecosystem. Science 3: $32-51.2011$.

Menezes, A. N., Figueiredo, J. L., Buckup, P. A., \& Moura, R. L. Catálogo das espécies de peixes marinhos do Brasil. Editora: Museu de Zoologia. São Paulo. 159p. 2003.

Militelli, M. I., Macchi, G. J., \& Rodrigues, K. Comparative reproductive biology of Sciaenidae family species in the Río de La Plata and Buenos Aires Coastal Zone, Argentina. Journal of the Marine Biological Association of the United Kingdom, 93(2): 413-423. 2013.

Ministério do Meio Ambiente. Instrução Normativa $n^{o}$ 53, de 22 de Novembro de 2005. Estabelece o tamanho mínimo de captura de espécies marinha e estuarinas do litoral sudeste e sul do Brasil. Diário Oficial da União, Brasília, 24 de novembro de 2005, n 225, Seção 1, 86-87 p. 2005.

Nóbrega, M. F., Lessa, R., \& Santana, F. M. Peixes marinhos da região nordeste do Brasil: Editora Martins \& Cordeiro. 208 p. 2009.

Nunes, Y. B. S., Silva, L. R., Freitas, J., \& Figueiredo, M. B. Estudo dos aspectos reprodutivos do Tchiurus lepturus (Linnaeus, 1758) na Costa Maranhense, Brasil. Brazilian Journal of Development, 6(1), 4201-4210 p., 2020.

Santos, P. R. S., Einhardt, A. C. M. C., \& Velasco, G. A pesca artesanal da miragaia (Pogonias cromis, Sciaenidae) no estuário da Lagoa dos Patos, Brasil. Boletim do Instituto de Pesca, 42(1): 89-101. 2017.

Santos, P. V. C. J., Almeida-Funo, I. C. S., Piga, F. G., França, V. L., Torres, S. A., \& Melo, C. D. P. Perfil socioeconômico de pescadores do município da Raposa, estado do Maranhão. Revista Brasileira de Engenharia de Pesca, 6 (1): I-XIV, 2011.

Santos, R. S., Silva, J. P. C., Costa, M. R., \& Araújo, F. G. O tamanho de primeira maturação como parâmetro para estabelecimento de tamanho mínimo de captura para corvina no sudeste do Brasil. Boletim do Instituto de Pesca, São Paulo, 41(3): 507-518, 2015.

Senguttuvan, M., \& Sivakumar, A. A. Studies on the Maturation and Spawning of Channa striata (Bloch, 1793). Journal of Aquaculture Tropics, 31: 13-23. 2016 .

Silva, C. E. L. Biologia reprodutiva do camurim, Megalops atlanticus, e do peixe espada, Trichiurus lepturus, duas das principais espécies de peixes ósseos capturados no extremo oeste do Ceará, Brasil. Dissertação. Universidade Federal do Ceará, 97 p. 2019.

Szpilman, M. Peixes marinhos do Brasil: guia prático de identificação. Instituto Ecológico Aqualung, 288p. Rio de Janeiro, 2000.

Tsikliras, A. C., \& Stergiou, K. I. Size at maturity of Mediterranean marine fishes. Reviews in Fish Biology Fishereries, 24: 219-268. 2014.

Vazzoler, A. E. A. Biologia da reprodução de peixes teleósteos: teoria e prática. Maringá: Eduem/SBI, Ed. da Universidade Estadual de Maringá e Sociedade Brasileira de Ictiologia. 169 p. 1996.

Véras, P. F., \& Almeida, Z. S. Biologia reprodutiva do Bagre bagre capturado pela pescaria de zangaria. Agrária, Recife, 11, 4, 2016. 